\title{
Sport hilft bei chronischen muskuloskelettalen Schmerzen
}

\author{
Slavko Rogan und Jan Taeymans
}

\author{
Es besteht Evidenz, dass bei Menschen mit chronischen muskuloskelettalen Schmerzen \\ das Trainieren über der Schmerzgrenze analgesierender wirkt als das Üben im schmerzfreien \\ Bereich. Um den Patienten ihre Angst vor den Bewegungsschmerzen zu nehmen, sollten \\ Physiotherapeuten gezielt Aufklärungsarbeit leisten und "Sicherheitshinweise“ einsetzen. \\ Für die Praxis eignet sich ein Stufenmodell, das Ausdauer- und Krafttraining kombiniert.
}

Chronischer Schmerz ist „ein Schmerz, welcher seit mindestens drei bis sechs Monaten besteht und die betroffene Person physisch, psychisch-kognitiv und sozial beeinträchtigt“ [14]. Es handelt sich um ein weltweites Phänomen, das den Lebens- und Berufsalltag der Betroffenen massiv einschränkt. Allein im deutschsprachigen Raum sind mehr als 25 Millionen Menschen betroffen [11]. Im Rahmen einer europaweiten Umfrage gaben 19\% von 46.394 Personen an, ihre Arbeitsstelle wegen chronischer Schmerzen verloren zu haben - rund ein Drittel von ihnen war in Vollzeit beschäftigt [5].

Gesundheitskosten Hohe Prävalenzzahlen führen zu hohen Gesundheitskosten. Die jährlichen Gesamtausgaben für Patienten mit chronischen Rückenschmerzen betragen in der Schweiz - in der Eidgenossenschaft leben 8,4 Millionen Menschen - rund 10,3 Milliarden CHF bzw. 9,12 Milliarden Euro [26].

\section{ARTEN CHRONISCHER SCHMERZEN}

Die aktuelle Version der ,Internationalen statistischen Klassifikation von Krankheiten und verwandter Gesundheitsprobleme، (ICD 11) der WHO umfasst die häufigsten klinisch relevanten Erkrankungen [24]. Je nach Schmerzart, welche die Krankheiten verursachen, wurden diese in sieben Gruppen eingeteilt:

1. primärer chronischer Schmerz

2. chronischer Krebsschmerz

3. chronischer posttraumatischer und postoperativer Schmerz

4. chronischer neuropathischer Schmerz

5. chronischer Kopfschmerz und orofazialer Schmerz

6. chronischer viszeraler Schmerz

7. chronischer muskuloskelettaler Schmerz

\section{Chronischer muskuloskelettaler}

\section{Schmerz}

Angesichts der hohen Prävalenzzahlen ist der chronische muskuloskelettale Schmerz für die Physiotherapie von besonderer Relevanz. In der Praxis stellen sich Therapeuten dann oftmals die Gretchenfrage, ob Sport als Therapieoption für die Betroffenen geeignet ist, und ob ein Training auch Schmerzen auslösen darf?

\section{CHRONISCHER MUSKULOSKELETTALER SCHMERZ \\ Der chronische muskuloskelettale Schmerz (CMS) ist ein anhaltender oder wiederkehrender Schmerz, welcher als Teil eines Krankheitsprozesses entsteht, der direkt Knochen, Gelenke, Muskeln oder ver- wandte Weichteilgewebe befällt [24].}

Für die Durchführung von Anamnese und objektiven Befund sowie für die Planung der Behandlungsstrategie von CMS-Patienten sind traditionelle Schmerzmodelle, welche eine Gewebeschädigung als exklusive Quelle für den nozizeptiven Input beschreiben, nicht ausreichend. Demgemäß müssen zusätzliche Faktoren, die einen chronisch muskuloskelettalen Schmerz modulieren können, unbedingt berücksichtigt werden [21]. Aktuell werden in der Literatur folgende vier Wirkmechanismen beschrieben.

\section{Zentrale Sensibilisierung}

Eine Zentrale Sensibilisierung wird von zellulären Veränderungen der Nozizeptoren im zentralen Nervensystem (ZNS) ausgelöst. Diese Veränderungen führen zur erhöhten Erregbarkeit für normale Reize [19]. 
Zentrale Sensibilisierung ist ein Überbegriff für Krankheitszeichen wie Hyperalgesie, Allodynie, zeitliche Schmerzsummierung oder die diffuse noxische inhibitorische Kontrolle (DNIC) [6]. DNIC bezieht sich auf einen endogenen Schmerzmodulationsweg, der oft als ,schmerzhemmend' beschrieben wird. Sie tritt auf, wenn die Reaktion auf einen schmerzhaften Reiz durch einen anderen, oft räumlich entfernten, schädlichen Reiz gehemmt wird.

\section{Immunsystem}

Bei einer Entzündung wird die unspezifische Immunabwehr aktiviert. In dieser Phase setzen verschiedene Zelltypen des infizierten Gewebes unterschiedliche Botenstoffe frei. Diese binden sich an Toll-Like-Rezeptoren (TLR) der Mikroglia des ZNS und können den Reiz, d. h. einen drohenden oder tatsächlichen Gewebeschaden, an afferente Neurone des Hinterhorns weiterleiten (Sensibilisierung) [10].

\section{Emotionen}

Angst, Depression und Katastrophisieren sind ebenfalls wichtige Faktoren zur Erklärung von CMS. Bewegungsangst kann durch eine verstärkte nozisensorische Weiterleitung auf Niveau des Rückenmarks, durch Modulierung der deszendierenden Bahnsysteme sowie durch zeitliche Summation die zentrale Sensibilisierung beeinflussen. Zeitliche Summation führt zur gesteigerten GlutaminSensitivität der Hinterhornneurone und folglich zur erhöhten nozisensorischen Wahrnehmung [22,23].

Eine Reduzierung katastrophisierender Gedankenspiele sowie eine physische Aktivitätserhöhung können die Wirkungsmechanismen verschiedener Behandlungsmaßnahmen erklären [16].

\section{Amygdala}

Mittels bildgebender Verfahren konnte Evidenz gesammelt werden, dass Angst vor Schmerzen die Amygdala überaktiviert und dadurch die zentrale Sensibilisierung und demzufolge auch chronische muskuloskelettale Schmerzen begünstigt [20].

Limbisches System Die Amygdala ist ein pariges Kerngebiet des Gehirns im medialen Teil des jeweiligen Temporallappens. Sie ist Teil des Limbischen Systems, einer Funktionseinheit des Kortex, die der Verarbeitung von Emotionen und der Entstehung von Triebverhalten dient. Einige Krankheiten lassen sich auf Störungen des Limbischen Systems oder der Amygdala zurückführen so vermutlich die Unfähigkeit, emotionale Situationen einschätzen zu können, Gedächtnisstörungen, Posttraumatische Belastungsstörungen, Narkolepsie, Autismus sowie Depressionen und Angststörungen.

\section{Schmerztherapie}

Für die Behandlung chronischer Schmerzpatienten empfiehlt die ,Deutsche Schmerzliga' eine angepasste und komplexe Therapie. Allerdings gibt es weder Patentrezepte noch Allheilmittel. Den meisten Erfolg verspricht eine multimodale Therapie, in welcher die Eigenaktivität des Patienten von größter Bedeutung ist. Die Eigenaktivität eines Menschen umfasst das aktive Bewegen sowie Sport in all seinen Variationen und Facetten - so auch den Gesundheitssport.

\section{Gesundheitssport}

\section{GESUNDHEITSSPORT}

„Das Spektrum des Gesundheitssports umfasst alle präventiven und rehabilitativen körperlichen Aktivitäten, wobei weniger die Leistung, sondern die Erhaltung, Förderung oder Wiederherstellung der Gesundheit im Vordergrund stehen." [25]

Studienlage Bewegungstherapeutische Maßnahmen bei CMS-Patienten sind prinzipiell erfolgsversprechend, aber je nach Bewegungsprogramm sind die Effektgrößen meistens moderat und weisen zudem eine große Variabilität auf [21]. Eine Metaanalyse mit sieben randomisiert kontrollierten Studien (insgesamt 385 Teilnehmer) ergab, dass ein Training mit Intensitäten über der Schmerzgrenze bei CMS-Patienten eine statistisch signifikant größere Schmerzreduktion im Vergleich zum Trainieren im schmerzfreien Bereich bewirkt (Standardisierte Mittelwertdifferenz, SMD = - 0,27; 95\%; Konfidenzintervall, Cl: $-0,54$ bis $-0,05)[6]$.

\section{Wirkmechanismen von Gesundheitssport}

Um das therapeutische Management von CMS-Patienten zu optimieren, ist es unbedingt notwendig, die Wirkmechanismen therapeutischer Reize zu verstehen. Eine jüngst publizierte Studie von Smith et al. beschreibt drei Mechanismen, warum ein Training über der Schmerzgrenze bei CMS-Patienten sinnvoll ist [21].

\section{Zentrale Schmerzprozesse}

Ein Training, dessen Intensität die Schmerzgrenze überschreitet, steigert Herzfrequenz und Blutdruck und führt über die arterielle Aktivierung von Barorezeptoren zur erhöhten Freisetzung von Beta-Endorphinen aus Hypophyse und Hypothalamus und aktiviert so das endogene OpioidSystem [1]. Der Hypothalamus kann durch die periaquäduktale graue Substanz das deszendierende nozisensorische, inhibitorische System anregen und so zum endogenen, analgetischen Effekt beitragen [18] (• Abb. 1). 


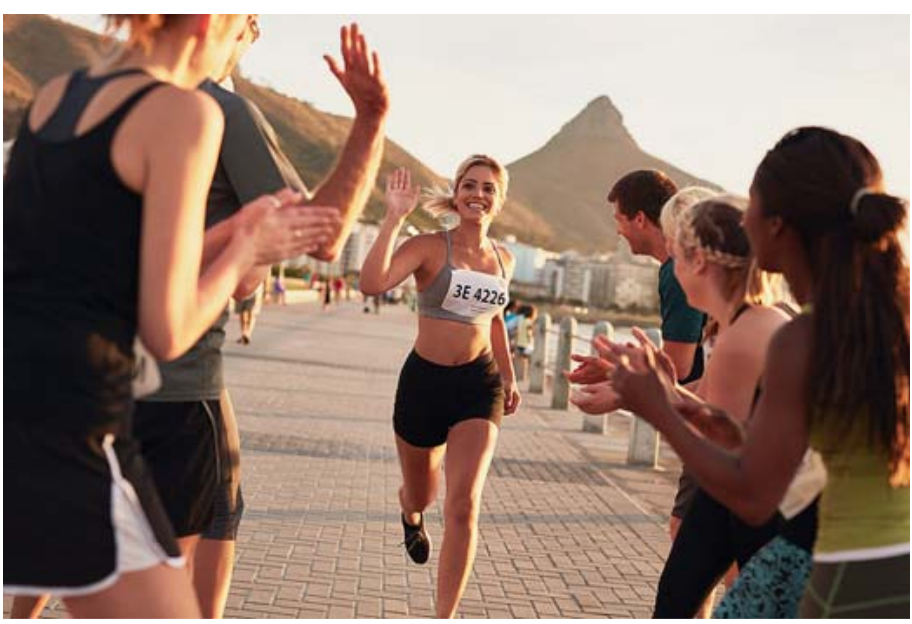

- Abb. 1 Beim so genannten „Runner’s High“ sorgen Endorphine dafür, dass Läufer trotz Erschöpfung und Schmerzen lange Distanzen durchhalten und sich dank der kleinen, Glücksbringer' auch noch gut dabei fühlen. Quelle: Jacob Lund/stock.adobe.vom (Symbolbild)

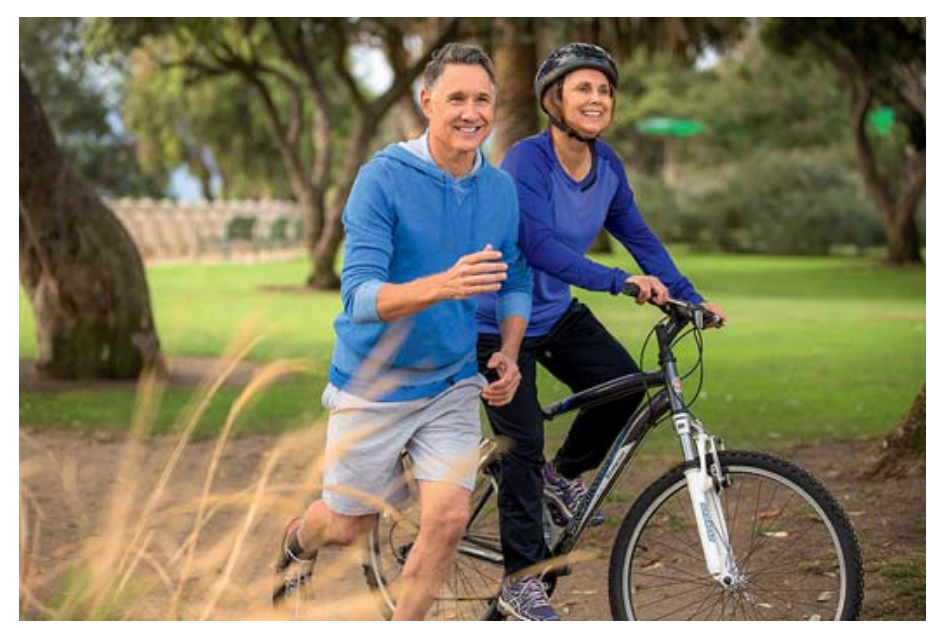

- Abb. 2 Um die richtige Sportart für einen Patienten zu identifizieren, kann die 3-pw-Regel helfen. Gemäß derer sollte die Sportart zum Patienten ,passen', für ihn ,praktikabel', ,präzise', durchführbar (Trainingsplan) sowie ,wirksam' sein. Quelle: elnariz/stock.adobe.com (Symbolbild).

\section{Immunsystem}

Gleichzeitig löst ein Training im schmerzhaften Bereich bei CMS-Patienten auch Stress aus. Dieser aktiviert die Amygdala und bedingt u.U. überschießende Entzündungsreaktionen des Immunsystems. Wird aber ein schmerzhaftes Training begleitet von angstreduzierenden Maßnahmen, so kann dies die Aktivität von Amygdala und Immunsystem sowie den Sympathikotonus stärker reduzieren als bei körperlicher Aktivität im schmerzfreien Bereich [12].

\section{Emotionale Aspekte}

Während eines schmerzhaften Trainings können „Sicherheitshinweise" des Therapeuten hilfreich sein, einem Patienten die Angst vor dem Bewegen im schmerzhaften Bereich zu nehmen bzw. diese zu senken. Es gibt Evidenz, dass schmerzhaftes Bewegen die Angstwahrnehmung reduzieren und folglich die Amygdala-Aktivität herabsetzen kann, wodurch nozisensorische, inhibitorische Systeme positiv beeinflusst werden $[15,17]$.

\section{„Bitte seien Sie beruhigt, es gibt keinen Schaden!“”}

Edukation In der Praxis können Physiotherapeuten ihren Patienten während des Trainings erklären, dass die Gelenkschmerzen durch mangelnde Bewegung bzw. eine Schonhaltung bedingt sind und nun die umliegenden Strukturen trainiert werden müssen, um dadurch die Muskelkraft und die Gelenkbeweglichkeit zu steigern.

\section{Implikationen für den klinischen Alltag}

Im Kontext des biopsychosozialen Modells hat Gesundheitssport eine wichtige soziale Bedeutung. Gesundheitssportlich aktive CMS-Patienten haben Kontakte zu anderen Menschen und wirken so selbstwirksam einer Vereinsamung - auch diese kann Schmerzen auslösen und triggern - entgegen.

Aus Sicht der Salutogenese sollen CMS-Patienten befähigt werden, ihre noch vorhandenen konditionellen Fähigkeiten zu erhalten und zu optimieren. Demgemäß ist ein Perspektivwechsel hin zum Patienten gefragt. Welche Art von Gesundheitssport am Ende gewählt wird, soll individuell entschieden werden ( $\bullet$ Abb. 2).

Studienlage Ein Cochrane-Review gab Auskunft über die Effekte von Sportarten und deren Trainingsmodalitäten bei erwachsenen CMS-Patienten [9]. Berücksichtigt wurden 21 Literaturübersichtsarbeiten mit insgesamt 381 Studien, in die 37.143 Teilnehmer involviert waren. Mit Ausnahme von lediglich drei Arbeiten zeigten die restlichen Studien (99,2\%) eine positive Wirkung auf den Schweregrad des Schmerzes. Neben der analgesierenden Wirkung von Sport entdeckten die Autoren auch einen positiven Effekt auf die Lebensqualität der Studienteilnehmer. Nur selten wurden unerwartete Nebenwirkungen bzgl. der Ausübung einer Sportart gemeldet. 


\section{Gesundheitssport für Patienten mit chronischen muskuloskelettalen Schmerzen}

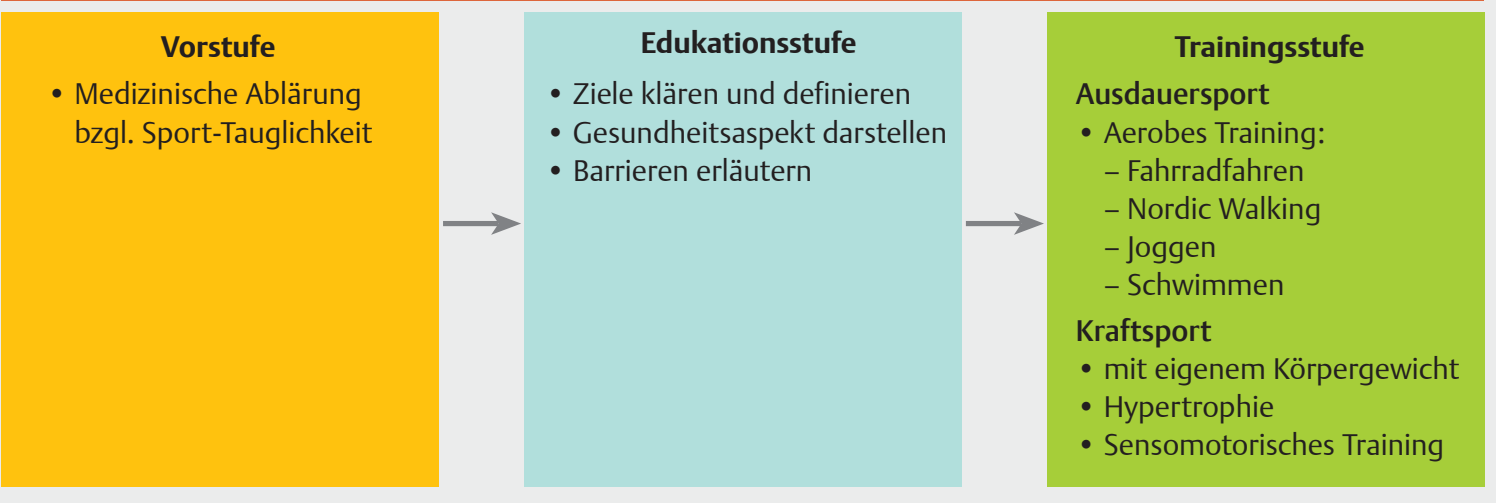

- Abb. 3 Stufenmodel für Gesundheitssport. Quelle: Slavko Rogan

- Tab. 1 Trainingsmodalitäten für Ausdauer- und Kraftsport (modifiziert nach [4]).

\begin{tabular}{|c|c|c|}
\hline & Ausdauersport & Kraftsport \\
\hline Frequenz & $\begin{array}{l}\geq 2 \times / \text { Woche } \\
\geq 6 \text { Wochen }\end{array}$ & $\begin{array}{l}2-3 \times / \text { Woche } \\
\geq 6 \text { Wochen }\end{array}$ \\
\hline Intensität & $\begin{array}{l}\text { " niedrige Intensität: } 50>55 \% \text { HRmax* } \\
\text { " moderate Intensität: } 55<70 \% \text { HRmax } \\
\text { " hohe Intensität: } 70<90 \% \text { HR max } \\
\text { " niedrige Intensität: RPE** } 8-10 \\
\text { " moderate Intensität: RPE } 11-13 \\
\text { - hohe Intensität: RPE } 14-16\end{array}$ & $\begin{array}{l}\text { - } \text { niedrige Intensität: RPE 8-10 } \\
\text { - } \text { moderate Intensität: RPE 11-13 } \\
\text { - hohe Intensität: RPE 14-16 } \\
\text { " niedrige bis moderate Intensität: 40-60\% 1WM*** } \\
\text { - hohe Intensität: bis } 90 \% 1 \mathrm{WM}\end{array}$ \\
\hline Dauer & $\begin{array}{l}\text { - } 20-60 \text { Minuten } \\
\text { - bei körperlicher Dekonditionierung:<20 Minuten }\end{array}$ & $\begin{array}{l}\text { - niedrige bis moderate Intensität: 1-2 Sätze mit } \\
\text { 15-20 Wiederholungen } \\
\text { - hohe Intensität: 1-2 Sätze mit 8-12 Wiederholungen }\end{array}$ \\
\hline Progression & $\begin{array}{l}\text { - bei Steigerung der Toleranz: von RPE 8-10 auf RPE 11-13 } \\
\text { - hochintensives Training: RPE } \geq 14 \\
\text { " die Trainingsdauer sollte vor der Trainingsintensität } \\
\text { erhöht werden }\end{array}$ & $\begin{array}{l}\text { - RPE 8-10 auf RPE } 11-13 \\
\text { - hochintensives Training: RPE } \geq 14 \\
\text { - die Anzahl der Wiederholungen sollte vor der } \\
\text { Trainingsintensität erhöht werden }\end{array}$ \\
\hline \multicolumn{3}{|c|}{${ }^{*}$ HRmax (Maximum heart rate) = maximale Herzfrequenz } \\
\hline \multicolumn{3}{|c|}{${ }^{* *}$ RPE (Rate of Perceived Exertion) = subjektive Anstrengung des Patienten (bewertet mit Borg-Skala) } \\
\hline \multicolumn{3}{|c|}{ *** $1 \mathrm{WM}=1$ Wiederholungsmaximum (entspricht beim Krafttraining einer Widerstandsgröße von 100\%) } \\
\hline
\end{tabular}

\section{Management von Gesundheitssport}

Um Menschen mit chronischen muskuloskelettalen Schmerzen zu motivieren, Gesundheitssport zu betreiben und so aktiv ihren Beschwerden gegenüberzutreten, eignet sich ein dreistufiges, aufeinander aufbauendes Vorgehen ( $\vee$ Abb. 3 ).

\section{Vorstufe}

Vor Aufnahme des Gesundheitssports sollten die ärztliche Abklärung eines kardio-vaskulären Risikos sowie eine profunde ganzheitliche medizinische Untersuchung des Patienten erfolgen. Im Zweifelsfall ist auch ein Belastungs-EKG angebracht.

\section{Edukationsstufe}

Es ist evident, dass im Vorfeld gesteckte Erwartungen die Behandlungsparameter Schmerz und Beeinträchtigung maßgeblich beeinflussen. Hohe Zielerwartungen eines Patienten wie bspw. die vollkommene Schmerzfreiheit wirken sich - wenn dieses Ziel nicht erreicht wird - auf den weiteren Therapie- bzw. Trainingsverlauf negativ aus [7]. Infolgedessen muss neben der spezifischen Trainingsberatung auch eine Aufklärung über mögliche Effekte von Gesundheitssport auf CMS erfolgen. 
Gleichzeitig ist bekannt, dass Angst, Panikattacken sowie katastrophisierende Gedanken stark mit Schmerzen und Beeinträchtigungen korrelieren [13]. Entsprechend ist es unerlässlich, diese Emotionen bereits vor Beginn eines Trainings zu identifizieren.

\section{Trainingsstufe}

In der Literatur werden für CMS-Patienten Ausdauersportarten (Gehen, Nordic Walking, Fahrradfahren, Joggen oder Tanzen), Kraftsport sowie Wassersport (Schwimmen, Aqua-Fitness) [2, 3, 8] empfohlen. Die Trainingsmodalitäten für den Ausdauer- und Kraftsport gestalten sich unterschiedlich ( $\bullet$ Tab. 1$)$.

\section{Zusammenfassung}

Traditionelle Schmerzmodelle sind für das Verständnis der Ätiologie sowie für die Befunderhebung und Planung einer Behandlungsstrategie von Patienten mit CMS nicht ausreichend. De facto müssen beitragende Faktoren wie die zentrale Sensibilisierung, die Emotionen des Patienten, sein Immunsystem sowie eine evtl. vorliegende Überaktivität der Amygdala beachtet werden.

Trainingsintensitäten über der Schmerzgrenze zeigen größere schmerzreduzierende Effekte im Vergleich zu einem Training im schmerzfreien Bereich. Die Aktivierung des endogenen Opioid-Systems, die Wirkung therapeutisch gezielt eingesetzter "Sicherheitshinweise“ sowie ein verbessertes Stressmanagement können diese Effekte erklären.

Der Gesundheitssport als Therapieansatz für CMS-Patienten umfasst die Stufen Vorabklärung, Edukation und Training. Das Training selbst ist eine Kombination aus Ausdauer- und Muskelkrafttraining.

\section{Autorinnen/Autoren}

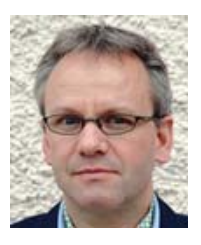

Prof. Dr. Slavko Rogan ist Dozent für den Studiengang Physiotherapie an der Berner Fachhochschule sowie Wissenschaftler. Sein Forschungsinteresse konzentriert sich auf die Entwicklung von Präventions- und Trainingsprogrammen, die Untersuchung der Wirksamkeit von Manueller Therapie und sensomotorischem Training.

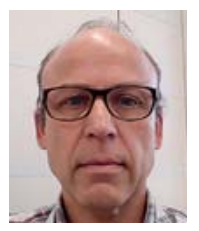

Prof. Dr. Jan Taeymans referiert an der Berner Fachhochschule, Sektion Gesundheit, zum Thema chronischer Schmerz im PhysiologieUnterricht. Zudem ist er Mitglied im wissenschaftlichen Beirat bei physioscience.
Korrespondenzadresse

Prof. Dr. Slavko Rogan

Murtenstr. 10

$\mathrm{CH}-3008$ Bern

slavko.rogan@bfh.ch

Literatur

[1] Bender T, Nagy G, Barna I et al. The effect of physical therapy on beta-endorphin levels. Eur J Appl Physiol 2007; 100 (4): 371-382. doi:10.1007/s00421-007-0469-9

[2] Bennell KL, Hinman RS. A review of the clinical evidence for exercise in osteoarthritis of the hip and knee. J Sci Med Sport 2011; 14 (1): 4-9. doi:10.1016/j.jsams.2010.08.002

[3] Bidonde J, Busch AJ, Webber SC et al. Aquatic exercise training for fibromyalgia. Cochrane Database Syst Rev 2014; 28 (10): CD011336. doi:10.1002/14651858.CD011336

[4] Booth J, Moseley GL, Schiltenwolf M et al. Exercise for chronic musculoskeletal pain: a biopsychosocial approach. Musculoskeletal Care 2017; 15 (4): 413-421. doi:10.1002/msc.1191

[5] Breivik H, Collett B, Ventafridda $V$ et al. Survey of chronic pain in Europe: prevalence, impact on daily life, and treatment. Eur J Pain 2006; 10 (4): 287-333. doi:10.1016/j.ejpain.2005. 06.009

[6] Chou R, Huffman LH. Medications for acute and chronic low back pain: a review of the evidence for an American Pain Society/American College of Physicians clinical practice guideline. Ann Intern Med 2007; 147 (7): 505-514

[7] Colloca L, Benedetti F. Nocebo hyperalgesia: How anxiety is turned into pain. Curr Opin Anaesthesiol 2007; 20 (5): 435439. doi:10.1097/ACO.0b013e3282b972fb

[8] Fransen M, McConnell S, Harmer AR et al. Exercise for osteoarthritis of the knee. Cochrane Database Syst Rev 2015; 1: CD004376. doi:10.1002/14651858.CD004376.pub3

[9] Geneen LJ, Moore RA, Clarke C et al. Physical activity and exercise for chronic pain in adults: an overview of Cochrane Reviews. Cochrane Database Syst Rev 2017 24; 4: CD011279. doi:10.1002/14651858.CD011279.pub3

[10] Guo LH, Schluesener HJ. The innate immunity of the central nervous system in chronic pain: the role of Toll-like receptors. Cell Mol Life Sci 2007; 64 (9): 1128-1136. doi:10.1007| s00018-007-6494-3

[11] Häuser W, Schmutzer G, Hinz A et al. Prävalenz chronischer Schmerzen in Deutschland. Schmerz 2013; 27 (1): 46-55. doi:10.1007/s00482-012-1280-z

[12] Hilz M], Devinsky O, Szczepanska H et al. Right ventromedial prefrontal lesions result in paradoxical cardiovascular activation with emotional stimuli. Brain 2006; 129 (Pt 12): 33433355. doi:10.1093/brain/awl299

[13] Manini TM, Hong SL, Clark BC. Aging and muscle: a neuron's perspective. Curr Opin Clin Nutr Metab Care 2013; 16 (1): 21-26. doi:10.1097/MCO.0b013e32835b5880

[14] Merskey HE. Classification of chronic pain: descriptions of chronic pain syndromes and definitions of pain terms. Pain Suppl 1986; 3: S1-226

[15] Milad MR, Quirk G]. Neurons in medial prefrontal cortex signal memory for fear extinction. Nature 2002; 420 (6911): 70-74. doi:10.1038/nature01138 
[16] Miles CL, Pincus T, Carnes D et al. Can we identify how programmes aimed at promoting self-management in musculoskeletal pain work and who benefits? A systematic review of sub-group analysis within RCTs. Eur J Pain 2011; 15 (8): 775. e1-11. doi:10.1016/j.ejpain.2011.01.01

[17] Morgan MA, Romanski LM, LeDoux JE. Extinction of emotional learning: contribution of medial prefrontal cortex. Neurosci Lett 1993; 163 (1): 109-113

[18] Nijs J, Kosek E, Van Oosterwijck J et al. Dysfunctional endogenous analgesia during exercise in patients with chronic pain: to exercise or not to exercise? Pain Physician 2012; 15 (3 Suppl): ES205-213

[19] Noble M, Treadwell JR, Tregear S] et al. Long-term opioid management for chronic noncancer pain. Cochrane Database Syst Rev 2010; (1): CD006605. doi:10.1002/14651858. CD006605.pub2.

[20] Simons LE, Moulton EA, Linnman C et al. The human amygdala and pain: evidence from neuroimaging. Hum Brain Mapp 2014; 35 (2): 527-538. doi:10.1002/hbm.22199

[21] Smith BE, Hendrick P, Bateman M et al. Musculoskeletal pain and exercise-challenging existing paradigms and introducing new. Br J Sports Med 2018; pii: bjsports-2017-098983. doi:10.1136/bjsports-2017-098983
[22] Sullivan M]L, Thorn B, Haythornthwaite JA et al. Theoretical perspectives on the relation between catastrophizing and pain. Clin J Pain 2001; 17 (1): 52-64

[23] Thacker M, Moseley L. Pathophysiological mechanisms of chronic pain. In: Corns I (Hrsg.) The routledge handbook of philosophy of pain, 1. Auflage. New York: Routledge; 2017

[24] Treede R-D, Rief W, Barke A et al. A classification of chronic pain for ICD-11. Pain 2015; 156 (6): 1003-1007. doi:10.1097| j.pain. 0000000000000160

[25] Urhausen A, Kindermann W, Dickhuth $\mathrm{H}$ et al. Trainingsempfehlungen im Gesundheitssport und Klassifikation der Sportarten. In: Kindermann W, Dickhuth H, Nieß A. (Hrsg.). Sportkardiologie: Körperliche Aktivität bei Herzerkrankungen. 2. Auflage. Steinkopff: Darmstadt; 2007

[26] Wieser S, Horisberger B, Schmidhauser S et al. Cost of low back pain in Switzerland in 2005. Eur J Health Econ 2011; 12 (5): 455-467. doi:10.1007/s10198-010-0258-y

Bibliografie

DOI https://doi.org/10.1055/a-0888-1940

Der Schmerzpatient 2019; 2: 120-125

(c) Georg Thieme Verlag KG Stuttgart · New York ISSN 2512-6210 